\title{
Does the "new philosophy" in predictive, preventive and personalised medicine require new ethics?
}

\author{
Eugenijus Gefenas • Asta Cekanauskaite • \\ Egle Tuzaite • Vilius Dranseika • Dainius Characiejus
}

Received: 16 March 2011 / Accepted: 30 March 2011 / Published online: 20 April 2011

(C) European Association for Predictive, Preventive and Personalised Medicine 2011

\begin{abstract}
This paper maps the ethical issues that arise in the context of personalised medicine. First, it highlights the ethical problems related to increased predictive power of modern diagnostic interventions. Such problems emerge because the ability to identify individuals or groups of individuals that can potentially benefit from a particular therapeutic intervention also raises a question of personal responsibility for healthrelated behaviour and lifestyle. The second major area of ethical concern is related to health prevention and distributive justice. The paper discusses the ethical challenges brought by the personalised medicine in the context of the Additional Protocol to the Convention on Human Rights and Biomedicine concerning Genetic Testing for Health Purposes. Finally, it notes that the issue of consent in the context of biobanks, the need to rethink the prevalent models of research designs and to communicate relevant findings to the donors of biological materials deserve further discussion.
\end{abstract}

Keywords Predictive diagnostics · Preventive healthcare . Personalised medicine $\cdot$ Medical ethics $\cdot$ Paternalism . Patient benefits and risks

D. Characiejus is a National Representative of EPMA in Lithuania.

E. Gefenas $(\bowtie) \cdot$ A. Cekanauskaite $\cdot$ E. Tuzaite $\cdot$ V. Dranseika Faculty of Medicine, Department of Medical History and Ethics, Vilnius University,

M.K.Ciurlionio Str. 21/27,

Vilnius LT 03101, Lithuania

e-mail: eugenijus.gefenas@mf.vu.lt

D. Characiejus

Faculty of Medicine, Vilnius University,

M.K.Ciurlionio Str. 21/27,

Vilnius LT 03101, Lithuania

D. Characiejus

Center for Innovative Medicine,

Zygimantu 9,

Vilnius, Lithuania

\section{Introduction}

According to the well-known 20th-century American physicist and philosopher of science Thomas Samuel Kuhn, from time to time sciences face "paradigm shifts". These "paradigm shifts" open up new approaches and bring remarkable changes to their development. It seems that the editorial of the first issue of The EPMA Journal marks a similar shift in medicine when it describes the paradigm change from delayed interventional nature of traditional medicine to the concept of predictive, preventive and personalised medicine [1]. For the sake of brevity, in the remainder of this article we will refer to this new concept of medicine as "personalised medicine". According to the editorial, this change marks a new philosophy in patient treatment as it moves away from a reactive approach of curing diseases that have already developed to a more proactive, preventive strategy to tackle human diseases before they start disrupting the functioning of a person. It seems that the philosophy of personalised medicine brings about messages, which can also be important from the ethical point of view. First, it expects the emerging and future diagnostic interventions to be much more accurate and therefore conducive to better treatment results due to both early detection of diseases and targeted therapeutic interventions on intervention-specific subgroups of patients. Second, personalised medicine also suggests to strengthen the patient's responsibility to follow the measures of disease prevention and to comply with the procedures of early interventions, which can help to cope with the disease in its early stage of development. Compliance with these measures is a crucial condition to achieve the mentioned benefits of more accurate diagnostics and more efficient therapeutic interventions. Last but not least, personalised medicine aims to develop preventive strategies to avoid the emergence of pathological conditions in the first place. 
These seem to be very much welcome developments. They can bring benefits both to the individuals who will be able to live long and productive lives and to the societies, which would not spend disproportionate amounts of resources to curative medicine, sometimes with only marginal effectiveness [2, 3]. Does this expected shift in the power of prediction and prevention, which is associated with the development of personalised medicine, raise any ethical concerns? Does the new philosophy in medicine direct us towards the new paradigm of medical ethics? These are the questions we will try to address in this paper.

\section{Personalised medicine and Hippocratic ethics}

Let us first turn to the second half of the 20th century, which marked the emergence of modern medical ethics or bioethics. One of the most important shifts in the history of medical ethics was the shift from traditional paternalism, which requires the physician to benefit his patient according to the best of his ability and judgement, to the ethics of personal autonomy and informed consent based on the dialogue between the physician and the patient and shared decision making. This shift has remarkably transformed the doctor-patient relationship a few decades ago. For example, cancer patients, who once were not informed about their disease in order to be protected from the disturbing news and psychological distress, now are equal partners in the decision making process concerning their treatment strategy. The latter is now regarded to be the ethically preferable strategy to be followed in all the societies that respect human rights [4].

The shift to autonomy-based ethics was influenced by at least two factors: the rise of the human rights movement, which has also affected the understanding of patients' rights; and the increasing role of chronic diseases in the general structure of human morbidity and mortality. The paternalistic character of the "medical expert", who is able to predict and unilaterally recommend a treatment strategy to save or prolong the life of his or her patient, was for many centuries a prevalent model of physician-patient relationship. This model, however, has nowadays become hardly compatible with a good clinical practice where prevalent causes of morbidity shifted from acute and infectious diseases towards the chronic diseases. In the context of treating chronic, neurodegenerative diseases or some types of cancer there is usually no single best strategy to choose. The choice here is not between the acceptance and decline of the life-saving treatment (which is one of the most important goals in the acute care medicine) but among several options neither of which offer an effective cure of the condition. In these circumstances, patient's preferences, values and world-views start to play a crucial role in the medical decision making and therefore lead to autonomy-based therapeutic relationship.

Another important feature of the traditional Hippocratic ethics is it's individualism: putting the benefits to the individual patient above the interests of the community. Modern medical ethics has challenged this fundamental feature of the Hippocratic tradition as well. Due to the fact that the cost of modern medicine has risen enormously during the last few decades, the rationing of scarce health care resources based on the effectiveness of health care interventions is becoming a necessary condition of the sustainable health care system, capable of providing equitable access to health care services to the citizens.

At this point we can link the main features of personalised medicine with the vocabulary of medical ethics. Increased power of prediction and greater certainty in some therapeutic choices can lead to more directive type of relationship between the doctor and the patient. For example, if the physician can distinguish between different types of cancer and knows that certain types of this disease are susceptible to certain types of therapy, which can entirely stop the malignant growth, ought not she or he use some stronger measures of persuasion and be more directive towards the non-compliant patients? Would not this look like some kind of paternalism? Secondly, should the personalised medicine develop preventive strategies for many diseases, would not the enforcement of compliance on the level of health care system be the most efficient strategy to implement the benefits of such strategies? Should not we think about some measures to increase patients' responsibility for their own health?

\section{Ethics of genetic testing}

Let us first examine those ethical concerns that are related to the increased power of prediction, which is supposed to be brought in the near future by the developments in the field of genetic testing and genetic screening. The importance of these issues has been reflected in the Additional Protocol concerning Genetic testing for Health Purposes to the Council of Europe Convention of Biomedicine and Human Rights of 2008 (further in the text refered to as "Additional Protocol") [5]. For the purposes of this paper we will concentrate on the following principles: clinical utility, respect for private life and the right to information (that also includes the right not to know), sharing of genetic information between the relatives, and non-directive manner of counselling. 
Clinical utility One of the most important criteria relevant to both practical implementation and ethical assessment of genetic testing is its clinical utility. Clinical utility is to be understood by the value of the test results in guiding the person concerned in his or her choices regarding prevention or therapeutic strategies [6]: para 57]. The clinical utility is mainly determined by two factors: predictive value of the test and the presence of preventive and therapeutic strategies related to the condition revealed by the test. The combination of these factors determines the clinical utility of the test and, at the same time, the appropriateness of offering the test to the individual. For example, the predictive value of the tests for monogenic disorders, e.g., Huntington disease (the late onset autosomal dominant severe neurological disorder), can be very high, however, therapeutic options are rather limited. Therefore, the offer of this test to the members of the family who can be the carriers of the pathological gene is a very sensitive one. Taking into account that genetic testing can be related to a very sensitive information and its preventive value can sometimes only be limited to reproductive choices of the affected individuals, it should be offered to the persons concerned with great caution.

The situation is even more complex if the predictive value of the test is not very high. Take, for example, the following hypothetical situation: a person undergoes a genetic test, which predicts a 25 percent chance of developing cardiovascular disease in the period of 20 years in case a person continues smoking, as compared to 10 percent chance of developing the disease if smoking is quitted. What is the practical importance and clinical utility of the information provided by such a test? Is the difference between these two situations significant enough to offer the test and consequently to build a preventive policy?

Respect for private life and the right to information In modern health care, patients are entitled to know any information about their health, including the results of genetic tests. However, our discussion on the criterion of clinical utility shows how complex the implementation of these rights can be in the context of predictive uncertainty and limited therapeutic choices. Therefore, the right to information is directly linked to two other important provisions recommended by the Additional Protocol: tests should be performed under (a) individualised medical supervision that is followed by (b) appropriate genetic counselling to prevent misinterpretation of the results and to soften psychological stress [5].

Wish not to be informed In the field of genetics the right to information is also linked to another principle - the wish of a person not to be informed about the results of the genetic test [5]: Art 16 Para 3]. This is a general principle applicable in the whole medicine. However, the wish of a person not to be informed in the context of genetic testing has its own specificity. Such a situation can arise when a patient, who has information based on the family history about a potentially dangerous health status, such as having a 50 percent chance to be a carrier of the Huntington's disease, prefers not to know the results of the genetic test concerning this disease carried out for some other purposes.

At the same time it should be acknowledged that sometimes the patient's right not to know might conflict with the doctor's duty to provide care [6]: Para 57]. It might happen that making a person knowledgeable about the results of a test is the only possibility to prevent the disease or delay its development. In some situations the right not to know can also be impracticable, e.g. prescribing certain treatment or recommending consulting a doctor of a special field may by itself reveal the presence of a disease. Even more controversial situation can arise if a person concerned does not want to know the results of the test that can have impact on the family members. This leads to another ethical dilemma, whether and under what circumstances the results of person's genetic test can be revealed to his or her family members.

Do the results of a genetic test belong to a person? Some authors believe that the development of personalised medicine can strengthen the family ties because the results of genetic tests can make people more responsible not only for their own health but also for their relatives' too. On the other hand, it is questionable whether genetic relations imply any special moral obligation to be responsible for other people [7].

Information about health and private life is considered to belong to the patient and therefore it should be kept confidential by the doctor who is normally only allowed to reveal this information to the third parties with the patient's consent. The most common exception to this rule is the situation when a non-disclosure can endanger life or health of the third party or the patient himself or herself. This situation can be also relevant in the field of genetics because the information provided by a genetic test can be not only important to a person who underwent a genetic testing but also to some of his/her family members. What if a patient does not agree to communicate this information to the relatives? Could the confidentiality of the patient be infringed in this case? Probably. The arguments will depend on the circumstances of a particular situation. Here we simply want to point out the collision between the doctor's duty to respect the patient's right to confidentiality and his or her commitment to safeguard the wellbeing of other people. The Additional Protocol recommends to inform a person about the importance for the relatives to have access to this information, and let him or her to make the decision 
[5]: Art 18]. However, the Protocol leaves it up to the national legislations to set up the requirements, criteria or circumstances for indicating situations allowing a doctor to reveal the information without person's consent. In some cases the courts both in Europe and the US enforce the option of sharing information between the relatives. For example, a court in New Jersey (US) ruled that the physician had a duty to directly warn the daughter of a patient about the risk to develop a colon cancer (which caused father's death 25 years ago), perhaps even over her father's objections [8].

Non-directive manner of counselling Finally, it seems to be important to stress still another provision of the Additional Protocol. Its Article 8 requires the genetic counselling to be given in a "non-directive manner" [5]. This requirement is an expression of the principle of respect for personal autonomy which was already presented as one of the fundamental principles of modern medical ethics. However, it should be taken into account that the idea of non-directive counselling as an attempt to avoid any influence or pressure on a person can be problematic in the context of personalised medicine. First, indirect pressure can occur because of a privileged position of a counsellor in terms of knowledge, experience and social status. Second, and more important for our discussion, the increased power of prediction, which is supposed to be achieved due to the developments of personalised medicine, can significantly increase this "authority" of a doctor giving advice to the patient. Especially sensitive situations of this kind can arrise with regard to reproductive choices that may include the termination of pregnancy.

Ethical problems that are encountered in the process of genetic testing will also be faced in relation to the screening programmes. The importance of clinical utility as well as such principles as respect for private life and the right to information, consent and genetic counselling in the context of genetic screening programmes is also recognised by the Additional Protocol [6]: Para 61]. The mentioned principles are also included into the list of criteria for genetic screening programmes of the UK National Screening Committee [9].

\section{Ethics of prevention}

Genetic testing enables both to adjust medical treatment to genetic characteristics of a patient and to predict the risks of developing certain diseases, which consequently can help a patient to take measures to prevent (or at least to delay or soften) the manifestation of the predicted illness. It is believed that the ability to detect a susceptibility for a particular illness before its manifestation and taking appropriate preventive actions can significantly lower mortality from certain diseases [10]. The move towards preventive policies promises remarkable improvements in health care delivery. However, it also raises ethical concerns related to the need of enforcing certain behaviors and life-styles. In this section we will concentrate on the policies that offer rewards or limitations of access to some types of health care services.

Access to health care in the context of personalised medicine Any consideration to impose sanctions for a certain health-related behavior or life-style is problematic due to a long-standing tradition of universal health care coverage. This tradition most probably has its roots in a strong feeling of solidarity, which encourages to provide access to health care irrespective of the ability to pay or other social factors. However, another reason not to place the responsibility on the shoulders of patients could be the complexity of differentiating between the conditions where a certain disease was a result of a genetic predisposition from those where the crucial factor in developing the disease was "unhealthy" life-style.

Personalised medicine seems to offer a distinction between these two factors and therefore weakens the background of the traditional conception of health care, which avoids imposing responsibility on patients for their health status. An additional factor, which has been challenging this traditional "responsibility free" access to health care, was a dramatic increase of expenditure on modern health care. For example, the U.S. government spent more than $\$ 2.3$ trillion on health care in 2008 (on average $\$ 7,681$ per person), more than three times the $\$ 714$ billion in 1990, according to the Kaiser Family Foundation [11]. In order to keep the portion of the GDP allocated to health care on approximately the same level, the countries are forced to introduce strategies to prioritise and ration health care services. In this context personalised medicine seems also to be contributing by developing tools to distinguish between high - and low-efficiency interventions to particular groups of patients, which is an important instrument for rationing scarce health care resources. This can require difficult allocation decisions to be faced by the physicians and patients in situations where the efficiency of treatment will be rather different in different groups of patients suffering from the same disease. For example, it has been already established that the treatment of a colorectal cancer with panitumumab and cetuximab is most effective for patients without particular mutations in the KRAS gene. However, even in this group patients do not show the same response to the drug-only some achieve the maximal gain of 2 years of life, whereas others gain just 2 months [12]. If it were possible to clearly distinguish the patients who fall into a particular group, would not this also affect the allocation decisions in a way that those 
gaining only two months of life would not be eligible for the treatment, especially if it is an expensive one?

Personal responsibility for health Having in mind the possible implications of personalised medicine both to the disease prevention and to the access to health care services debate, let us now turn to the central ethical issue of prevention-personal responsibility for one's own health. Are the obese, smoking, drinking, or physically not sufficiently active persons responsible for their ill health? Should there be any sanctions for those who do not comply with the rules of "healthy lifestyle", which is commonly associated with proper weight, non-smoking, keeping the appointments and following the advice of a doctor? However, these are not entirely new issues in the health care debate. As has been pointed out earlier, the progress in genetic prediction raises their ethical relevance and reveals new complexities. For example, if a genetic test helps to distinguish between genetic and nongenetic (social) factors contributing to the development of the disease and reveals that a person has a 50 percent chance to develop a colonic cancer, should the policy makers make an attempt to create incentives or even force a person to choose a certain life-style if it is known that certain measures (e.g., diet, exercising, quitting-smoking, regular check-up) can prevent the development of the disease? What would happen if the disease would develop and it would be known that a person hadn't complied or only partly complied with the "prevention plan" that has been offered? Would this also mean that the public funds shouldn't be used for the treatment of such a person or that he or she should pay part of the price of the treatment?

Enforcing personal responsibility Although nowadays policies to make people responsible for their own health seems to be quite rare, there have recently been several attempts to implement the idea of personal responsibility for health in practice. For example, some Primary Care Trusts (UK) refuse to perform hip or joint surgery or even any routine operation for smoking patients or patients with BMI exceeding 30. In Germany, patients who keep their checkup schedule can pay less for their dental care than those who do not. At the same time, the financial incentives are also offered for those who are considered to comply with the health maintenance measures, such as participating in age-related health promotion or screening programmes. Depriving insured persons of a right to free treatment of complications arising from "lifestyle" related choices (such as cosmetic surgery, tattoos, or piercing) have been regarded as one of the most controversial elements of the German reform [13].

Another example of enforcing responsibility for health comes from the U.S. The West Virginia Medicaid programmes covering health care services for low income population was redesigned to reduce basic services for most healthy children and adults, but gave an opportunity to get more benefits if they agree to sign and comply with the provisions of a "Medicaid Member Agreement". The Agreement says that "To remain in the enhanced plan, members must keep their medical appointments, receive screenings, take their medications, and follow health improvement plans. Members whose benefits are to be reduced because they have not met these criteria will receive advance notice and have the right to appeal. Those who meet their health goals will receive "credits" that will be placed in a "Healthy Rewards Account" to be used for purchasing services that are not covered by the Medicaid plan" [14].

Although these practices might look attractive from the first sight, they raise some difficult questions of how justifiable the measures that enforce so-called healthy behavior are. How far can the governments and the health care providers go in enforcing people to live "healthy" life? Are people responsible for being obese or drug/alcohol addicts or are they just the victims of their social environment, conditions in the family, educational background? Even taking into account that a right to accessible health care is not an absolute right, it seems that the discussed cases might bring some suspicion concerning possible discrimination based on person's genetic characteristics, health condition or life style at least as far as these factors in many cases are still not clearly separable. Most of national and international guidelines, legal acts and policy documents dealing with patients' rights or specifically genetic data, stress the importance of genetic privacy and necessity of safeguards against discrimination on genetic grounds [5]: Art 4; [15]: Art 7].

This does not, however, mean that all the measures of putting more responsibility on the people for their own health contradict human solidarity and right to selfdetermination. For example, taking into account that missing appointments with the GPs and nurses can seriously disrupt the provision of health care services (e.g. English patients missed 11 million appointments with GPs in 2006), we can be rather positive about the appeals to patients to take seriously their responsibilities by cancelling appointments in time [13]. It seems that the tendency of putting more responsibility on the shoulders of patients will be even more strengthened in the context of personalised medicine. However, we have to be aware that "the more one moves away from the incentivefocused end of the spectrum to the disincentive/penalty end, the higher the risk of increasing unfairness and potential "victim-blaming" [13] of already vulnerable populations.

\section{Research ethics issues in personalised medicine}

The development of personalised medicine is based on intensive scientific research which by itself raises many ethical issues. Let us mention several of them. The 
development of screening programmes and targeted individualised treatment options for specific types of diseases fuelled a new wave of research on human biological materials. National genome projects and disease-oriented biobanks are being established all over the world and increasing attention is paid to the legal and ethical frameworks that regulate this type of research. The problem is that in this field of research the idea of specific consent, which is relevant to clinical research protocols with clearly defined aims, design and plan of interventions, could hardly be applicable since in large-scale genome projects and biobanks it is not possible to envisage in detail all potential future uses of biological samples. Some countries have already developed new legislation which allows to meet these new challenges. To mention but a few examples, the provisions of broad consent were implemented in Estonia and the UK, which run population-based genome projects, as well as in Norway, which has recently included the provisions of "broad consent" into its research legislation [16]: Ch 4, Sec 14]. Similar tendencies can also be observed at the European level in the Council of Europe Recommendation on Research on Biological Materials of Human Origin of 2006 [17]. The Explanatory Memorandum to this instrument has introduced a distinction between the interventions that aim at removing materials for use in a specific research project from those interventions where the sample is removed for research storage. The latter option is combined with a broader type of consent, which does not require to describe specific aims of research projects for which the biological sample can be used in the future [18]. The Recommendation Rec(2006)4 and some other documents also introduce the idea of so-called multi-layered or "tiered" consent [19], which enables donors to choose from several options ranging form allowing to use his or her biological sample only in one particular disease-specific research project to allowing the sample to be used for all possible future research.

Communicating the incidental findings of genetic testing obtained during the biobanking research to the donor is another issue which raised ethical concerns due to the tension between researcher's obligation to protect confidentiality of research participants and his or her commitment to provide feedback on accidental health related findings to them [20]. There has been so far no agreement on this issue. On the one hand, a possibility of the feedback of health-related information to the research participants is an attractive offer, which can encourage people to take part in the research [19]. On the other hand, the participant's wish to know the results of genetic testing can endanger protection of his or her personal data since sharing of information involves more risks to "leak" the information to the third parties and to infringe the confidentiality. In addition, this question raises concerns related to the clinical utility of the test and possible psychological harm these results can cause if they are presented to the patient without a proper genetic counselling.

These are not the only research ethics issues raised in the context of personalised medicine. For example, there have been some important insights about the need to change prevalent study designs in cancer research and to move from large-scale clinical trials to small comparative trials based on groups of patients who have different predictive biomarkers. The problem is that in large scale research, such as phase III clinical trials, the subgroups of patients who do not benefit from new therapeutic interventions can suffer a detrimental effect of the investigational therapy. This can be prevented if the study on predictive biomarkers is introduced in the early phases of research and these biomarkers are used to differentiate between treatmentresistant and treatment-efficient patients. What is important to our discussion, the early studies that reveal the predictive biomarkers can lead us to rethink the choice of the comparative groups. Traditional approach to this type of research has been to avoid a comparative group that receives no active treatment. However, given a possibility of cancer-promoting or low-efficiency effect of an anticancer intervention, a group of untreated patients might be seen as an acceptable choice [21] in the context of scientific research in the field of personalised medicine.

\section{Concluding remarks}

The promises and aspirations of personalised medicine are well-taken and could indeed in the foreseeable future lead to what has been described as the paradigm change in health care. However, increased predictive power of medicine and targeted therapeutic interventions are not only promising more efficient treatment for patients, improved quality of longer human lives and effective use of scarce health care resources. This paradigmatic change also requires to take a fresh look on the ethical and social implications of the new medicine. One of the central ethical issues emerging in this context is increasing role of personal responsibility for health, which is an understandable consequence of the shift from reactive disease-treatment oriented medicine towards the proactive approach of preventive medicine with an emphasis on personal responsibility for health. At the moment the health care systems of many countries are only making their first steps towards the balancing of the traditional solidarity-based medicine with the new ethos of patient responsibility for his or her better health prevention. However, the slip to the "victimblaming" scenario, where vulnerable people are deprived of treatment arguing that they are responsible for their poor health, while the factors that contributed to it are beyond their control [13], is to be avoided. 
The emphasis on prediction and prevention can also affect the individual physician-patient relationship. The role of the health care practitioner as a neutral moderator providing the non-directive counselling in the context of personalised medicine, which emphasizes the measures of prevention and offers high levels of prediction, can also be questioned. It seems that a temptation towards a more directive approach in respect to non-compliant patients can be observed. However, such an approach should not infringe on patients' rights, which, among others, include the right to privacy and selfdetermination. Physician-patient relationship can also be affected by the emerging differentiation of patients into different groups according to their resistance/efficiency with regard to the treatment interventions. It will not be an easy choice to the doctor to tell a patient that a particular treatment is not available due to the fact that its efficiency does not reach a certain threshold.

Finally, developing personalised diagnostic techniques in the free-market societies can raise some other problems as well. We are already facing a rapid introduction of directto-consumer genetic testing into the market. On the one hand, it shifts the responsibility for some important health care choices to the patients and increases their selfdetermination. On the other hand, this process leaves space for detrimental consequences such as the risk of psychological harm and misinterpretation of the test since it is difficult to make sure that direct-to-consumer predictive genetic testing is followed by individualised medical supervision and genetic counselling.

Conflict of interest None declared.

\section{References}

1. Costigliola V. Preface. EPMA J. 2010;1:1-2.

2. Golubnitschaja $\mathrm{O}$. Time for new guidelines in advanced diabetes care: Paradigm change from delayed interventional approach to predictive, preventive and personalized medicine. EPMA J. 2010;1:3-12.

3. George B, Cebioglu M, Yeghiazaryan K. Inadequate diabetic care: global figures cry for preventive measures and personalized treatment. EPMA J. 2010;1:13-8.
4. Gefenas E. Bioethics, professional codes and law In: Pegoraro R, Putoto G, Wray E, editors. Hospital based bioethics: European perspective. Padova: Piccin; 2007:1-17.

5. Council of Europe. Additional protocol to the Convention on human rights and biomedicine, concerning genetic testing for health purposes. 2008. http://conventions.coe.int/Treaty/en/ Treaties/html/203.htm. Accessed 13 Mar 2011.

6. Council of Europe. Explanatory report to the Additional protocol to the Convention on human rights and biomedicine, concerning genetic testing for health purposes, 2008. http://conventions.coe. int/Treaty/EN/Reports/Html/203.htm. Accessed 13 Mar 2011.

7. Van Delden J. Tailor made pharmacotherapy: future developments and ethical challenges in the field of pharmacogenomics. Bioethics. 2004;18:303-21.

8. Safer v.Pack, 677 A.2d 1188 (N.J. App.), appeal denied, 683 A 2 d 1163 (N.J. 1996). Cited in: Clayton EW. Ethical, legal and social implications of genomic medicine. N Engl J Med. 2003;349:562-9.

9. UK National Screening Committee. Criteria for appraising the viability, effectiveness and appropriateness of a screening programme. 2011. http://www.screening.nhs.uk/criteria. Accessed 13 Mar 2011.

10. Roberts R. Personalized medicine: a reality within this decade. J Cardiovasc Trans Res. 2008;1:11-6.

11. Khan $\mathrm{H}$. Why health care costs keep rising: what you need to know. Abc News. 2010. http://abcnews.go.com/Politics/HealthCare/healthcare-costs-biggest-drivers/story?id=10044091. Accessed 13 Mar 2011.

12. Fleck LM. Personalised medicine's ragged edge. Hastings Cent Rep. 2010;40:16-8.

13. Schmidt H. Health responsibility, the Left, and the Right. The Bioethics Forum. 2007. http://www.thehastingscenter.org/ Bioethicsforum/Post.aspx?id=440. Accessed 13 Mar 2011.

14. Steinbrook R. Imposing personal responsibility for health. Engl J Med. 2006;355:753-6.

15. UNESCO. International declaration on human genetic data. 2003. http://portal.unesco.org/en/ev.php-URL_ID=17720\&URL_DO= DO_TOPIC\&URL_SECTION=201.html. Accessed 13 Mar 2011.

16. Parliament of Norway. Act No 44 on medical and health research. 2008. http://www.regjeringen.no/upload/HOD/HRA/Helseforskning/ Helseforskningsloven \%20-\%20ENGELSK \%20endelig\%2029\% 2006\%2009.pdf. Accessed 13 Mar 2011.

17. Council of Europe. Recommendation Rec(2006)4 of the Committee of Ministers to Member States on research on biological materials of human origin. 2006. https://wcd.coe.int/wcd/ViewDoc.jsp? id=977859. Accessed 13 Mar 2011.

18. Council of Europe. Draft explanatory memorandum to the Draft recommendation $\operatorname{Rec}(2006) 4$ of the Committee of Ministers to Member States on research on biological materials of human origin. 2006. https://wcd.coe.int/ViewDoc.jsp?id $=961161 \&$ Site $=$ COE. Accessed 13 Mar 2011.

19. Maschke K. Wanted: human biospecimens. Hastings Cent Rep. 2010;40:21-3.

20. Hedgecoe AM. Context, ethics and pharmacogenetics. Stud Hist Philos Biol Biomed Sci. 2006;37:566-82.

21. Characiejus D, Hodzic J, Jacobs J. "First do no harm" and the importance of prediction in oncology. EPMA J. 2010;1:369-75. 